\title{
Qualidade de vida em idosas: a importância da dimensão subjetiva
}

\author{
Quality of life in elderly women: the importance \\ of the subjective dimension
}

\author{
Tatiana Quarti IRIGARAY \\ Clarissa Marceli TRENTINI ${ }^{2}$
}

\begin{abstract}
Resumo
Este estudo teve por objetivo investigar o conceito de qualidade de vida para idosas, pontuando os aspectos que elas consideram mais importantes, os que melhoram e os que prejudicam sua qualidade de vida. Participaram do estudo 103 idosas, que responderam acerca de informações sociodemográficas e a um questionário composto por quatro questões abertas que investigavam sobre a sua percepção subjetiva de qualidade de vida. Utilizou-se o método amostral de conveniência. Os dados foram categorizados mediante análise de conteúdo. Os resultados demonstraram uma associação entre qualidade de vida e afetos positivos, saúde e independência. Os fatores apontados como importantes para qualidade de vida foram afetos positivos, saúde e boas condições financeiras. Prejudicam o bem-estar os fatores sociais, os problemas de saúde e familiares. Melhoraria a qualidade de vida ter mais saúde, lazer e menos problemas familiares. Conclui-se que a qualidade de vida é determinada por fatores múltiplos, contemplando aspectos físicos, psicológicos e sociais.
\end{abstract}

Unitermos: Envelhecimento. Idoso. Qualidade de vida.

\begin{abstract}
The aim of the presentstudy was to analyze the concept of quality of life in elderly women, demonstrating what is important for them, as well as those aspects that may improve or detract from their quality of life. The sample consisted of 103 elderly women that answered questions concerning socio-demographic information and a questionnaire consisting of four open questions about their subjective perception of quality of life. The Convenience sampling method was used. The data were categorized according to analysis of the content. The results showed an association between the concept of quality of life and positive affects, health and independence. The factors identified as being important to quality of life were positive affects, health and a good financial situation. The aspects that adversely affected their quality of life were social situation, health problems and family problems. The following aspects may improve their quality of life: Better health, more leisure time and fewer family problems. The study indicates that quality of life is determined by multiple factors, involving physical, psychological and social aspects.
\end{abstract}

Uniterms: Aging. Aged. Quality of life.

\footnotetext{
$\boldsymbol{\nabla \nabla \nabla \nabla}$

1 Pontifícia Universidade Católica do Rio Grande do Sul, Programa de Pós-Graduação em Gerontologia Biomédica. Av. Ipiranga, 6690, Jd. Botânico, 90610-000, Porto Alegre, RS, Brasil. Correspondência para/Correspondence to: T.C. IRIGARAY. E-mails: <tati.irigaray@ig.com.br>; <tatiana.irigaray@superig.com.br>.

2 Universidade Federal do Rio Grande do Sul, Cursos de Graduação e Pós-Graduação em Psicologia. Porto Alegre, RS, Brasil.
} 
A avaliação da qualidade de vida tem recebido a atenção de diferentes áreas do conhecimento nos últimos anos. Embora ainda inexista um consenso sobre o seu significado, há uma certa unanimidade entre os estudiosos em utilizar a definição do Grupo de Qualidade de Vida da Organização Mundial de Saúde (Grupo WHOQOL). De acordo com este grupo, a qualidade de vida é definida como a percepção que o indivíduo tem sobre a sua posição na vida, considerando o contexto de sua cultura e de acordo com os sistemas de valores da sociedade em que vive, bem como em relação aos seus objetivos, expectativas, padrões e preocupações (WHOQOL Group, 1998).

A qualidade de vida é um conceito difícil de ser medido, pois representa uma avaliação única, individual (Carr \& Higginson, 2001; Fry, 2000). Além disso, os valores de referência do indivíduo podem mudar com o passar do tempo (Carr, Gibson \& Robinson, 2001). Segundo o estudo de Bowling (1995), os domínios considerados importantes para a qualidade de vida mudam com a idade. Desta forma, pessoas mais jovens dariam mais importância ao trabalho e às finanças, enquanto os idosos julgariam a saúde e a mobilidade como os itens mais importantes para a qualidade de vida (Bowling, 1995).

Especificamente na velhice, a preocupação com a qualidade de vida ganhou expressão nos últimos trinta anos. Isto se deu, entre outros motivos, devido ao aumento do número de idosos na população e a expansão da longevidade. Sabe-se que a qualidade de vida compreende um conceito complexo, que tem múltiplas dimensões, é multideterminado, diz respeito à adaptação de indivíduos e grupos de pessoas em diferentes épocas da vida, de uma ou várias sociedades (Neri, 2005) Assim, definir qualidade de vida na velhice não é tarefa fácil, pois tanto a velhice quanto a qualidade de vida são eventos dependentes do tempo (Neri, 2000).

Da mesma forma, Lawton (1991) também sustenta a ideia da multidimensionalidade da qualidade de vida na velhice e acrescenta que ela seria realizada a partir de critérios intrapessoais e socionormativos, a respeito do sistema pessoa-ambiente de um indivíduo, no momento atual, no passado e no futuro. Para ele, a qualidade de vida na velhice é dependente de muitos elementos que estão inter-relacionados, sendo o produto de uma história interacional que iria se deli- neando à medida que os indivíduos e sociedades se desenvolvem. Ela envolveria uma comparação entre critérios objetivos e subjetivos associados a normas e a valores sociais e individuais, os quais estariam igualmente sujeitos a alterações no decorrer do tempo. 0 modelo de Lawton (1983) propõe que a avaliação da qualidade de vida na velhice dá-se sobre quatro dimensões sobrepostas e inter-relacionadas: condições ambientais, competência comportamental, qualidade de vida percebida e bem-estar subjetivo ou psicológico, das quais dependeria a funcionalidade do idoso.

De modo complementar, Neri (2003) sustenta que o conceito de qualidade de vida na velhice tem associação direta com a existência de condições ambientais que permitem aos idosos desempenhar comportamentos adaptativos, com a qualidade de vida percebida e também com o senso de autoeficácia. A avaliação subjetiva que cada indivíduo faz sobre seu funcionamento é o conteúdo primário da qualidade de vida percebida. Já a autoeficácia é um importante precursor do comportamento em todas as idades, uma vez que significa a crença aprendida do indivíduo a respeito de que dispõe dos recursos necessários para obter os resultados que almeja.

Ainda assim, apesar das definições acima, que incluem a ideia de subjetividade e/ou percepção pessoal, existem poucos estudos qualitativos sobre o significado da qualidade de vida para idosos (Borglin, Edberg \& Hallberg, 2005). Entre os publicados, há um destaque para a importância dos vínculos familiares, relações sociais, saúde, independência, bem-estar psicológico, papéis sociais, atividades, recursos financeiros, viver em casa e ter uma boa vizinhança para uma boa qualidade de vida na velhice (Leung, Wu, Lue \& Tang, 2004; Puts et al., 2007). Borglin et al. (2005) demonstraram que a qualidade de vida de idosos depende da preservação do self e da manutenção de objetivos ao longo de toda a vida. De acordo com esse trabalho, a experiência de qualidade de vida envolveria valores de vida, experiências anteriores, capacidade de se adaptar a mudanças, independência, atividade, saúde, relações sociais e viver em uma casa.

Em relação ao caráter subjetivo, atualmente reconhece-se que a qualidade de vida é uma avaliação dependente da subjetividade do indivíduo (WHOQOL Group, 1998). Segundo Trentini, Xavier e Fleck (2006) há 
uma grande variabilidade entre as pessoas em relação às suas capacidades de enfrentamento às limitações físicas e às doenças, e também às suas expectativas em relação aos aspectos de saúde. Os conceitos individuais podem influenciar de maneira determinante a percepção do indivíduo sobre o seu estado de saúde e a sua satisfação com a vida. Consequentemente, duas pessoas com o mesmo estado objetivo/real de saúde podem apresentar qualidade de vida totalmente diferentes, devido aos aspectos subjetivos. Desta forma, entende-se por subjetividade a percepção do indivíduo acerca de sua própria qualidade de vida, não contemplando aspectos objetivos da saúde da pessoa.

Estudos brasileiros sobre a percepção subjetiva de qualidade de vida de idosos também são poucos. Conforme Xavier, Ferraz, Marc, Escosteguy e Moriguchi (2003), em um estudo realizado com idosos em Veranópolis (RS) sobre a percepção subjetiva de qualidade de vida, a presença de doenças e as limitações físicas foram os fatores determinantes de uma qualidade de vida negativa. Por outro lado, a qualidade de vida positiva foi definida, principalmente, pela presença de saúde, atividade, relações familiares, relações sociais, renda e bens. Outro estudo com idosos na cidade de Botucatu (SP) mostrou a presença de três grupos distintos de respostas sobre o que é qualidade de vida. O primeiro grupo enfatizou as relações pessoais, o bem-estar psicológico e a saúde, priorizando a questão afetiva e a família. O segundo ressaltou hábitos saudáveis, lazer e bens materiais, enfatizando o prazer e o conforto. Já o terceiro grupo mencionou a espiritualidade, o trabalho, a retidão, a caridade, o conhecimento e ambientes favoráveis, identificando como qualidade de vida conseguir colocar em prática o seu ideário de vida (Vecchia, Ruiz, Bocchi \& Corrente, 2005).

Browne et al. (1994) realizaram uma pesquisa com idosos saudáveis, com mais de 65 anos, residentes na comunidade, buscando identificar quais os domínios de qualidade de vida mais relevantes para eles. Os autores verificaram que as variáveis mais destacadas foram as seguintes: atividades sociais e de lazer (95\%), saúde (91\%), relações com a família (89\%), condições de moradia (80\%) e religião (75\%).

Em outro estudo realizado com idosos na cidade de Porto Alegre (RS), cujo objetivo era identificar os fatores que permitem uma boa qualidade de vida na velhice, verificou-se que o domínio saúde foi o aspecto mais importante. Além da saúde, os idosos também ressaltaram os domínios psicológico, nível de independência, relações sociais, meio ambiente, espiritualidade e crenças pessoais (Fleck, Chachamovich \& Trentini, 2003).

Este estudo teve como objetivo geral investigar o conceito de qualidade de vida para idosas. Os objetivos específicos incluíram: a) averiguar os aspectos mais importantes para a qualidade de vida; b) investigar os aspectos que prejudicavam o bem-estar; e c) identificar os aspectos que melhorariam a qualidade de vida.

\section{Método}

\section{Participantes}

Tratou-se de um estudo transversal e a amostra foi composta por 103 idosas, com idades entre 60 e 86 anos, participantes da Universidade para a Terceira Idade (UNITI) da Universidade Federal do Rio Grande do Sul (UFRGS). O processo de amostragem foi o de conveniência, sendo que as 153 integrantes da UNITI foram convidadas a participar do estudo, mas apenas 103 consentiram em fazê-lo.

A UNITI é um projeto de extensão, ensino e pesquisa do Instituto de Psicologia da UFRGS, no Brasil. Esse projeto iniciou suas atividades no primeiro semestre de 1991, estando, atualmente, no seu 170 ano de funcionamento. Seu trabalho fundamenta-se nos princípios da educação permanente, tendo como objetivo principal potencializar os recursos humanos constituídos pela faixa etária dos sessenta anos ou mais. Seus participantes são pessoas da comunidade desta faixa etária, em média 150 participantes por semestre, em sua grande maioria mulheres idosas. Sua dinâmica de trabalho aborda questões cognitivas, sociais e comunitárias, levando os idosos a pensar e repensar o seu papel enquanto ser produtor e colaborador na sociedade. Na UNITI, os idosos são incentivados a conquistar, manter e preservar a autonomia, uma boa qualidade de vida e a independência. Também são estimulados a adquirir novas informações e ampliar conhecimentos. 


\section{Instrumentos}

A ficha de dados sociodemográficos levantou as seguintes variáveis: sexo, idade, estado civil, escolaridade, renda, ocupação atual e percepção de saúde.

A percepção subjetiva da qualidade de vida foi examinada por meio de um questionário composto por quatro perguntas abertas que investigaram: 1) o que é qualidade de vida; 2) o que é importante para a qualidade de vida; 3) aspectos que prejudicam o seu bem-estar; e 4) aspectos que melhorariam sua qualidade de vida. A questão 1 investigava sobre o conceito, ou seja, a definição de qualidade de vida para as idosas. A questão 2 averiguava os aspectos mais importantes para a qualidade de vida, podendo os mesmos estar inclusos ou não no conceito de qualidade de vida. Já as questões 3 e 4 buscavam identificar os aspectos que melhorariam ou piorariam a qualidade de vida. Essas questões foram motivadas pelo trabalho de Fleck et al. (2003), que investigou, igualmente, a partir da metodologia dos grupos focais, a percepção de idosos saudáveis e doentes acerca de qualidade de vida e bem-estar.

\section{Procedimentos}

Foram realizados contatos com a Universidade para a Terceira Idade (UNITI), procedendo-se, assim, à inclusão das participantes na amostra. Todas as idosas preencheram o Termo de Consentimento Livre e Esclarecido e, a partir daí, foram convidadas a responder acerca de informações sociodemográficas e percepção subjetiva de qualidade de vida.

Tanto a ficha de dados sociodemográficos quanto as questões sobre qualidade de vida são de caráter autoadministrável; contudo, um auxiliar de pesquisa (estudante de psicologia) sempre esteve à disposição das idosas para qualquer esclarecimento. As aplicações dos instrumentos foram feitas em grupos de até 15 participantes, sendo que cada participante respondeu individualmente aos dois questionários.

O projeto foi devidamente examinado e aprovado pelo Comitê de Ética em Pesquisa da Pontifícia Universidade Católica do Rio Grande do Sul (PUCRS), Porto Alegre, RS, Brasil (no 06/0309). A submissão do projeto ao Comitê de Ética e Pesquisa da PUCRS deu-se em função da execução da coleta paralelamente à elaboração de uma monografia para o Curso de Especialização em Psicologia Clínica: Avaliação Psicológica da UFRGS e de uma dissertação de mestrado realizada no Programa de Pós-Graduação em Gerontologia Biomédica da PUCRS.

A descrição dos dados sociodemográficos foi realizada por meio das frequências absolutas e relativas, bem como média e desvio-padrão. Para análise desses dados, foi utilizado o programa Statistical Package for the Social Sciences (SPSS) para ambiente Windows, versão 13.0.

As respostas das quatro questões abertas do questionário sobre o conceito de qualidade de vida, fatores importantes e aspectos que prejudicariam ou melhorariam a qualidade de vida foram analisadas mediante análise de conteúdo (Bardin, 2004).

Adotou-se o critério semântico para a identificação de categorias temáticas, baseadas no agrupamento de elementos de significados mais próximos que compunham as respostas das participantes. Após isso, foi realizada a contagem da frequência final dos elementos nas categorias. As repostas foram analisadas e, posteriormente, foram calculadas as frequências das categorias obtidas.

\section{Resultados e Discussão}

A amostra foi composta por 103 mulheres idosas com idades entre 60 e 86 anos (média de 69,2; desvio-padrão de 6,46). A maioria delas era aposentada (73,8\%) e percebia-se como saudável (81,6\%). A Tabela 1 mostra

Tabela 1. Características sociodemográficas da amostra estudada $(n=103)$. Porto Alegre (RS), 2006.

\begin{tabular}{lll}
\hline Variáveis & $\mathrm{n}$ & $\%$ \\
\hline Estado civil & & \\
Casada/com companheiro & 32 & 31,0 \\
Viúva & 46 & 44,7 \\
Separada/divorciada/solteira & 25 & 24,3 \\
Escolaridade & & \\
Ensino fundamental & 21 & 20,4 \\
Ensino médio & 40 & 38,8 \\
Ensino superior & 42 & 40,8 \\
Renda & & \\
1 a 5 salários-mínimos & 22 & 21,3 \\
6 a 10 salários-mínimos & 40 & 38,8 \\
11 a 20 salários-mínimos & 22 & 28,2 \\
mais de 20 salários-mínimos & 12 & 11,7 \\
\hline
\end{tabular}


as características sociodemográficas da amostra em estudo. Conforme pôde ser observado, o estado civil mais frequente foi o de viúva (44,7\%), a escolaridade mais prevalente o ensino superior $(40,8 \%)$ e a renda mais presente foi a de 6 a 10 salários-mínimos (38,8\%).

Na Tabela 2, estão apresentadas as respostas às questões sobre o conceito e os aspectos importantes para qualidade de vida. Verifica-se que o conceito de qualidade de vida apareceu associado com ter alegria na vida, amor, amizade, ter saúde, independência, atividades intelectuais, bom relacionamento com a família, boas condições financeiras, realizar atividades físicas, dieta equilibrada, conviver com a natureza, ser generosa e solidária. Essa evidência está de acordo com a literatura (Fleck et al., 2003; Lawton, 1983; Neri, 2005). No estudo de Fleck et al. (2003), o conceito de qualidade apareceu relacionado a uma heterogeneidade de aspectos, evidenciando a ideia de que a qualidade de vida de idosos constitui um construto multidimensional. Da mesma forma, Lawton (1983) e Neri (2005) afirmam que a qualidade de vida na velhice é um evento que tem múltiplas dimensões e é multideterminado. Ela consistiu em uma avaliação que contém várias dimensões, fundamentada em critérios sociais, normativos e do próprio indivíduo acerca das relações presentes, passadas e futuras entre o idoso e o seu ambiente.

O fator mais associado com qualidade de vida na velhice foi ter alegria na vida, amor e amizade. Esta associação foi encontrada no estudo de Fleck et al. (2003) como resultado do grupo focal realizado com idosos saudáveis, com idades entre 60 e 80 anos. Segundo os idosos daquele estudo, a qualidade de vida é definida como ter alegria na vida, amor e amizade. Em outro estudo realizado no interior de São Paulo com 365 idosos, cujo objetivo era investigar o conceito de qualidade de vida, verificou-se a existência de três grupos de respostas, sendo que um deles associava qualidade de vida com a questão afetiva e relações familiares (Vecchia et al., 2005).

Segundo os resultados do estudo aqui descrito, as idosas consideraram como itens mais importantes para a qualidade de vida os afetos positivos (ter alegria na vida, amor e amizades) e a saúde. Esse resultado concorda com a percepção de diversos trabalhos sobre a importância da afetividade, dos relacionamentos e da saúde para os idosos (Bowling, 1995; Browne et al., 1994). Alguns autores defendem a ideia de que as relações sociais são um dos componentes mais importantes da boa qualidade de vida de idosos, tanto quanto o estado de saúde (Farquar, 1995; Gabriel \& Bowling, 2004; Wilhelmson, Andersson, Waern \& Allebeck, 2005). É possível que estas duas variáveis estejam presentes de forma relativamente dependente, já que melhores condições de saúde auxiliam ou, ao menos, não prejudicam a busca pelas relações sociais ou vice-versa. Novaes (1997) também defende que as relações sociais são de extrema importância para a saúde física e mental do idoso, já que o isolamento social poderia ocasionar o surgimento da angústia e da solidão.

Conforme citado anteriormente, além dos afetos positivos e da saúde, outro aspecto que foi apontado como importante para a qualidade de vida foi o recurso financeiro favorável. Uma hipótese de explicação para

Tabela 2. Frequência de respostas às questões sobre qualidade de vida: definição e aspectos importantes para a qualidade de vida. Porto Alegre (RS), 2006

\begin{tabular}{lcclcc}
\hline Definição de qualidade de vida & $\mathrm{n}$ & $\%$ & O que é importante para qualidade de vida & $\mathrm{n}$ & $\%$ \\
\hline Alegria na vida, amor, amizade & 109 & 29,3 & Ter alegria na vida, amor, amizade & 117 & 33,4 \\
Saúde & 86 & 23,0 & Ter saúde & 26,7 \\
Independência, atividades intelectuais & 64 & 17,2 & Boas condições financeiras & 33 \\
Bom relacionamento com a família & 37 & 10,0 & Independência, atividades intelectuais & 33 & 9,5 \\
Boas condições financeiras & 37 & 10,0 & Bom relacionamento com a família & 33 \\
Realizar atividades físicas, ter uma dieta equilibrada, & 29 & 7,8 & Realizar atividades físicas, ter uma dieta equilibrada, & 31 & 8,5 \\
conviver com a natureza & & & conviver com a natureza & 8 \\
Ser generosa, solidária & 10 & 2,7 & Ser generosa, solidária & 2,3 \\
\hline Total & 372 & 100,0 & Total & 349 \\
\hline
\end{tabular}


este achado poderia estar no fato de que os idosos que têm melhores condições financeiras possuem mais acesso a bens e serviços. Em concordância com este resultado, Neri (1993) afirma que o recurso financeiro tem um efeito compensatório sobre as perdas da velhice, pois ajuda no acesso à saúde, ao lazer, à sensação de autoestima e à autoeficácia, tornando possível a compensação de perdas inevitáveis nessa fase da vida. Outros estudos encontraram, da mesma maneira, que a segurança financeira, o suporte de familiares e amigos e a independência são os principais determinantes de uma vida satisfeita para idosos (Irwin, 1999; Milne \& Williams, 2000).

Conforme pode ser observado (Tabela 3), os aspectos que mais prejudicam o bem-estar das idosas desta amostra são os seguintes: a política, a falta de segurança e as desigualdades sociais. Esse resultado demonstra que as idosas estão atentas aos problemas sociais e que se preocupam com a situação atual do país, demonstrando-se capazes de avaliar e opinar sobre o que interfere em seu bem-estar. Os problemas de saúde apareceram como o segundo item que mais prejudica o seu bem-estar. Uma hipótese de explicação para esse achado seria a de que um estado de saúde mais precário refletiria em diminuição da autonomia e da independência.

Segundo Pavarini e Neri (2000), o principal desafio que a longevidade impõe é a preservação da qualidade de vida frente às ameaças de restrição da autonomia e da independência. Da mesma forma, Lemos e Medeiros (2002) afirmam que a autonomia e a independência são ótimos indicadores de saúde entre idosos. A autonomia é o objetivo principal de todo ser humano: depender dos outros na idade adulta é algo rejeitado e temido.
O aspecto mais ressaltado pelas participantes para a melhoria da qualidade de vida foi ter melhores condições de saúde (Tabela 3). Uma associação entre qualidade de vida e saúde tem sido encontrada em diversos estudos (Bowling, 1995; Bowling et al., 2003; Fleck et al., 2003; Gabriel \& Bowling 2004; Puts et al., 2007; Tsang, Liamputtong \& Pierson, 2004; Xavier et al., 2003). ConformeTsang et al. (2004), em uma pesquisa realizada com idosos chineses, a saúde foi destacada como o aspecto mais importante para a determinação da qualidade de vida. Salientaram também a independência, afirmando que a mesma está diretamente relacionada com o estado de saúde e o desempenho de habilidades funcionais. Assim, a saúde, a independência e as habilidades funcionais seriam fatores determinantes de uma boa qualidade de vida.

A saúde não foi considerada o item mais significativo do conceito de qualidade vida e nem foi destacada como o aspecto mais importante para a qualidade de vida; entretanto, foi ressaltada como o aspecto mais importante para melhorá-la. Uma possibilidade de explicação para o referido achado estaria nas características sociodemográficas da amostra estudada. A amostra foi composta, em sua maioria, por idosas saudáveis. Essa característica poderia explicar o fato de que, por serem idosas saudáveis, desejam continuar tendo mais saúde para poderem melhorar cada vez mais a sua qualidade de vida. Segundo Puts et al. (2007), as pessoas idosas saudáveis e doentes diferem quanto a fatores importantes para a qualidade de vida. Assim, enquanto os doentes afirmaram que o contato social era o fator mais importante, os saudáveis atribuíram à saúde a maior importância.

Tabela 3. Frequência de respostas sobre o que prejudica o bem-estar e o que melhoraria a qualidade de vida. Porto Alegre (RS), 2006.

\begin{tabular}{lcclcc}
\hline Prejudica o seu bem-estar & $\mathrm{n}$ & $\%$ & Melhoraria a sua qualidade vida & $\mathrm{n}$ & $\%$ \\
\hline Política, falta de segurança, desigualdade social & 57 & 34,2 & Ter mais saúde & 26 & 20,0 \\
Problemas de saúde & 43 & 25,8 & Ter mais oportunidades de lazer e atividades físicas & 23 & 17,9 \\
Problemas familiares & 37 & 22,0 & Menos problemas familiares, mais amor por parte & 22 & 17,1 \\
& & & da família & 22 & 17,1 \\
Situação financeira desfavorável & 15 & 9,0 & Melhor situação financeira & 19 & 14,7 \\
Solidão & 10 & 6,0 & Menos violência, mais paz e igualdade & 17 & 13,2 \\
Nada & 5 & 3,0 & Nada, já tem uma boa qualidade de vida & 100,0 \\
\hline Total & 167 & 100,0 & Total & 129 & 10 \\
\hline
\end{tabular}


Faz-se importante ressaltar que tal estudo foi conduzido com uma amostra com características relativamente homogêneas, apresentando nível de escolaridade elevado e boa situação socioeconômica, o que pode não refletir a realidade dos idosos em geral. Entretanto, conhecer os aspectos importantes para a qualidade de vida de idosos pode ajudar na oferta de serviços e na implementação de políticas públicas e educacionais que levem em conta as necessidades e as especificidades desta faixa etária.

\section{Considerações Finais}

Os resultados obtidos evidenciaram que o conceito de qualidade de vida na velhice é composto por critérios tanto subjetivos quanto objetivos, sendo influenciado pelos valores do indivíduo e da sociedade em que ele vive. Os resultados corroboram estudos anteriores, demonstrando uma associação entre qualidade de vida e ter alegria na vida, amor, amizade, ter saúde, independência, atividades intelectuais, bom relacionamento com a família, boas condições financeiras, realizar atividades físicas, dieta equilibrada, conviver com a natureza, ser generosa e solidária. A ideia de que qualidade de vida é um construto multidimensional ficou evidente.

Observou-se que os afetos positivos (ter alegria na vida, amor e amizade) foram as variáveis mais citadas e associadas como determinantes importantes da qualidade de vida na velhice. Os aspectos evidenciados como mais prejudiciais ao bem-estar foram a política, a falta de segurança e as desigualdades sociais. As idosas também salientaram ter saúde como o item mais importante para a melhora da qualidade de vida.

Apesar de crescentes, ainda são poucos os estudos sobre a avaliação subjetiva da qualidade de vida feita por idosos. Desta forma, sugere-se o desenvolvimento de trabalhos que busquem identificar como o idoso percebe a sua qualidade de vida, seus aspectos importantes e prejudiciais, para que se possa implementar ações que resultem em melhora de sua qualidade de vida.

\section{Referências}

Bardin, L. (2004). Análise de conteúdo. Lisboa: Edições 70.
Borglin, G., Edberg, A. K., \& Hallberg, I. R. (2005). The experience of quality of life among older people. Journal of Aging Studies, 19 (2), 201-220.

Bowling, A. (1995). The most important thing in life. Comparison between older and younger populations age group by gender. Results from a national survey of the publics judgement. International Journal of Health Sciences, 6 (12), 169-175.

Bowling, A., Gabriel, Z., Dykes, J., Dowding, L. M., Evans, O., Fleissig, A., Banister, D., \& Sutton, S. (2003). Let's ask them: a national survey of definitions of quality of life and its enhancement among people aged 65 and over. International Journal of Aging \& Human Development, 56 (4), 269-306.

Browne, J. P., O'Boyle, C. A., McGee, H. M., Joyce, C. R. B., McDonald, N. J., O'Malley, K., \& Hiltbrunner, B. (1994). Individual quality of life in the health elderly. Quality of Life Research, 3 (4), 235-244.

Carr, A. J., Gibson, B., \& Robinson, P. G. (2001). Measuring quality of life: is quality of life determined by expectations or experience? British Medical Journal, 322 (7296), 1240-1243.

Carr, A. J., \& Higginson, I. J. (2001). Are quality of life measures patient centred? British Medical Journal, 322 (7298), 1357-1360.

Farquar, M. (1995). Elderly people's definitions of quality of life. Social Science and Medicine, 41 (10), 1439-1446.

Fleck, M. P. A., Chachamovich, E., \& Trentini, C. M. (2003). Projeto WHOQOL-OLD: método e resultado de grupos focais no Brasil. Revista de Saúde Pública, 37 (6), 793-799.

Fry, P. S. (2000). Whose quality of life is it anyway? Why not ask seniors to tell us about it? International Journal of Aging and Human Development, 50 (4), 361-383.

Gabriel, Z., \& Bowling, A. (2004). Quality of life from the perspectives of older people. Ageing and Society, 24 (5), 675-691

Irwin, S. (1999). Late, life, inequality and sociological theory. Ageing and Society, 19 (Part 6), 691-715.

Lawton, M. P. (1983). Environment and other determinants of well-being in older people. Gerontologist, 23 (4), 349-357.

Lawton, M. P. (1991). A multidimensional view of quality of life in frail elderly. In J. E. Birren, J. E. Lubben \& J. C. Rowe, D. E. Deutchmann (Eds.), The concept and measurement of quality of life in the frail elderly (pp.3-27). San Diego, CA: Academic Press.

Lemos, N., \& Medeiros, S. L. (2002). Suporte social ao idoso dependente. In E. V. Freitas, L. Py, A. L. Neri, F. A. C. Cançado, M. L. Gorzoni \& S. M. Rocha (Eds.), Tratado de geriatria e gerontologia (pp.892-897). Rio de Janeiro: Guanabara Koogan.

Leung, K., Wu, E., Lue, B., \& Tang, L. (2004). The use of focus groups in evaluating quality of life components among elderly Chinese people. Quality of Life Research, 13 (1), 179-190. 
Milne, A., \& Williams, J. (2000). Meeting the mental health needs of older women: taking social inequality into account. Ageing and Society, 20 (6), 699-723.

Neri, A. L. (1993). Qualidade de vida no adulto maduro: interpretações teóricas e evidências de pesquisa. In A. L. Neri (Org.), Qualidade de vida e idade madura (pp.9-55). Campinas: Papirus.

Neri, A. L. (2000). Qualidade madura no atendimento domiciliário. In Y. A. O. Duarte \& M. J. D. Diogo (Orgs.), Atendimento domiciliar: um enfoque gerontológico (pp.33-47). São Paulo: Atheneu.

Neri, A. L. (2003). Qualidade de vida na velhice. In J. R. Rebelatto \& J. R. S. Morelli (Orgs.), Fisioterapia geriátrica (pp.9-34). São Paulo: Manole.

Neri, A. L. (2005). Palavras-chave em gerontologia. Campinas: Alínea.

Novaes, M. H. (1997). Psicologia da terceira idade: conflitos possíveis e rupturas necessárias. Rio de Janeiro: NAU.

Pavarini, S. C. I., \& Neri, A. L. (2000). Compreendendo dependência, independência e autonomia no contexto domiciliar: conceitos, atitude e comportamentos. In Y. A. O. Duarte \& M. J.D. Diogo (Eds.), Atendimento domiciliar: umenfoquegerontológico (pp.49-70). São Paulo: Atheneu.

Puts, M. T., Shekary, N., Widdershoven, G., Heldens, J., Lips, P., \& Deeg, D. J. (2007). What does quality of life mean to older frail and non-frail community-dwelling adults in the Netherlands? Quality of Life Research, 16 (2), 263-277.
Trentini, C. M., Xavier, F. M. F., \& Fleck, M. P. A. (2006). Qualidade de vida em idosos. In M. A. M. P. Parente (Org.), Cogniçãoe envelhecimento (pp.19-29). Porto Alegre: Artmed.

Tsang, E. Y. L., Liamputtong, P., \& Pierson, J. (2004). The views of older Chinese people in Melbourne about their quality of life. Ageing and Society, 24 (1), 51-74.

Vecchia, R. D., Ruiz, T., Bocchi, S. M., \& Corrente, J. E. (2005). Qualidade de vida na terceira idade: um conceito subjetivo. Revista Brasileira de Epidemiologia, 8 (3), 246-252.

Xavier, F. M. F., Ferraz, M. P. T., Marc, N., Escosteguy, N. U., \& Moriguchi, E. H. (2003). Elderly people's definitions of quality of life. Revista Brasileira de Psiquiatria, 25 (1), 31-39.

Wilhelmson, K., Andersson, C., Waern, M, \& Allebeck, P. (2005). Elderly people's perspectives on quality of life. Ageing and Society, 25 (4), 585-600.

World Health Organization. (1998). The World Health Organization quality of life assessment (WHOQOL): development and general psychometric properties. Social, Science and Medicine, 46 (12), 1569-85.

Recebido em: 9/4/2007

Versão final reapresentada em: 2/4/2008

Aprovado em: 8/7/2008 